\title{
Improved Insulin Pharmacokinetics Using a Novel Microneedle Device for Intradermal Delivery in Patients with Type 2 Diabetes
}

\author{
Efrat Kochba, MD, Yotam Levin, MD, Itamar Raz, MD, and Avivit Cahn, MD ${ }^{2,3}$
}

\begin{abstract}
Background: Currently available short-acting insulin analogs have slower absorption compared with endogenous insulin occasionally resulting in immediate postprandial hyperglycemia. Intradermal (ID) injection facilitates faster drug absorption and may result in improved insulin pharmacokinetics.

Methods: Seventeen patients with type 2 diabetes were included in this single-center, pilot, open-label crossover study. Patients received 0.2 U/kg Insulin aspart ID injections using a MicronJet (MJ) needle and subcutaneous (SC) injections, using a conventional needle in a crossover design. Thirteen patients were studied under fasting conditions and four before a standard meal test. The pharmacokinetic/pharmacodynamic (PK/PD) profile, as well as the safety and tolerability of injections, was compared.

Results: Fourteen patients completed the study per-protocol. ID versus SC injection demonstrated significantly shorter Tmax (median 35 vs. 87.5 min $[P<0.001]$ ), while the Cmax did not significantly differ (median 80 vs. $55 \mu \mathrm{U} / \mathrm{mL}[P=0.085])$. Median insulin area under the curve (AUC; $360 \mathrm{~min}$ ) did not differ between the groups (9914 vs. 10,936 $\mu \mathrm{U} / \mathrm{mL} / \mathrm{min}[p=0.077]$ ), yet 0-60 min insulin AUC was higher with ID versus SC injection (mean \pm SD $3821 \pm 1429$ vs. $2534 \pm 737 \mu \mathrm{U} / \mathrm{mL} / \mathrm{min}[p=0.01]$ ) and $4-6 \mathrm{~h}$ AUC was lower with ID versus SC injection (mean \pm SD $2054 \pm 858$ vs. $2929 \pm 1412 \mu \mathrm{U} / \mathrm{mL} / \mathrm{min}[p=0.02]$ ). The relative bioavailability of the ID versus the $\mathrm{SC}$ insulin $\left(\mathrm{AUC}_{\mathrm{ID}} / \mathrm{AUC}_{\mathrm{SC}}\right.$ ) was similar (median 0.91 [95\% confidence interval 0.73-1.27]).

Conclusions: ID insulin injection delivered through an MJ needle demonstrated superior PK profile compared with conventional SC administration, including shorter Tmax and higher early and lower late exposure in patients with type 2 diabetes. This may help achieve better insulin coverage of meals and lower postprandial glucose excursions.
\end{abstract}

\section{Introduction}

I NSULIN REMAINS THE most effective blood glucose-lowering agent. ${ }^{1}$ Short-acting insulin analogs exhibit rather slow absorption kinetics with a Tmax of about 45-70 min, which is much longer than that of endogenic insulin in a healthy individual. ${ }^{2}$ Matching peak insulin levels to peak postprandial glucose excursions requires delivery of the insulin injection before the meal and assessment of portion sizes before eating. This poses some limitation on the spontaneity of eating and caution is needed to prevent hypoglycemia induced by too early insulin injection or ingestion of a smaller meal size than anticipated. Moreover, the relatively delayed insulin absorp- tion may lead to high glucose levels in the first 90-120 min after a meal, resulting in inadequate glycemic control.

To address the challenge of expediting insulin absorption and shortening the Tmax, some investigational approaches are being developed. ${ }^{3-5}$ These include reformulation of the substance injected, that is, by the addition of EDTA, hyaluronidase, or nicotinamide and arginine, or by employment of physical methods to enhance insulin absorption such as local heating of the injection site and intradermal (ID) or inhaled delivery of insulin. ${ }^{4}$

ID delivery is commercially used for several indications, including vaccines (BCG, influenza), local anesthesia, and aesthetics, as well as allergy and TB testing. ${ }^{6,7}$ The dermis is

\footnotetext{
${ }^{1}$ NanoPass Technologies Ltd., Nes Ziona, Israel.

${ }^{2}$ Diabetes Unit, Division of Internal Medicine, Hadassah Medical Organization, Jerusalem, Israel.

${ }^{3}$ Endocrinology and Metabolism Service, Division of Internal Medicine, Hadassah Medical Organization, Jerusalem, Israel.

(c) Efrat Kochba, et al. 2016; Published by Mary Ann Liebert, Inc. This Open Access article is distributed under the terms of the Creative Commons Attribution Noncommercial License (http://creativecommons.org/licenses/by-nc/4.0/) which permits any noncommercial use, distribution, and reproduction in any medium, provided the original author(s) and the source are credited.
} 


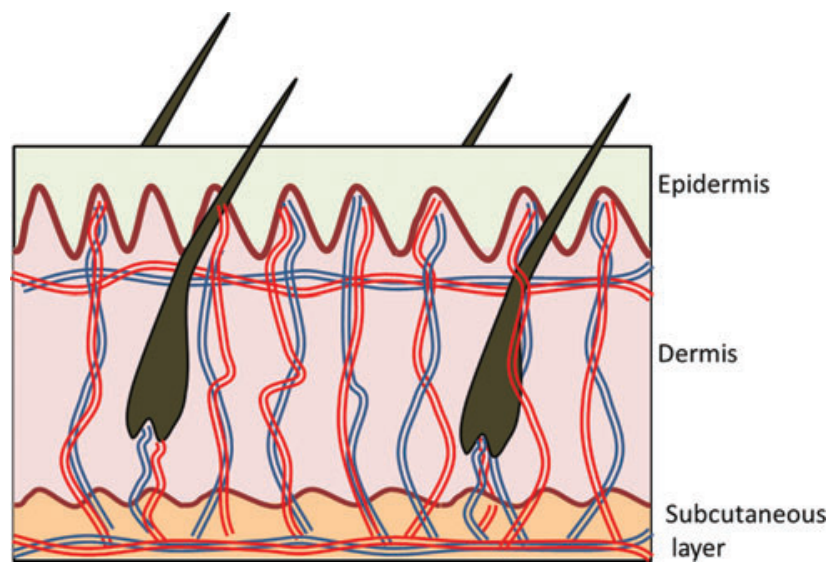

FIG. 1. An illustration of the extensive vascular network and arterial-venous shunts in the dermis.

highly vascularized (Fig. 1), thereby facilitating faster drug absorption. ID delivery of vaccines is currently done using a standard metal needle (technique known as Mantoux), which requires significant expertise. The challenge of using a standard needle to directly target the dermis without injecting too deep into the subcutaneous (SC) space or leaking externally, both frequently occurring, has limited the widespread use of ID injection. 8

Microneedles have been developed to facilitate reliable ID administration routes, which due to their minute size enable targeting the formulation injected into the dermis with maximal accuracy. MicronJet (MJ; NanoPass Technologies Ltd) is a microneedle device comprising four microneedles, each $0.45 \mathrm{~mm}$ in length, mounted on a standard syringe instead of a conventional needle (Fig. 2). Unlike the regular needle and syringe used for ID injection (Mantoux technique), the MJ device requires minimal expertise for successful ID injection, causes minimal pain during insertion, and potentially reduces the chances of trauma associated with needle handling. The safety and efficacy of ID delivery using the device were demonstrated in multiple clinical trials. ${ }^{10-14}$ Local adverse reactions, including local edema and erythema of the skin at the injection site, have been frequently observed and are typical of ID delivery of vaccines; these injection site reactions are usually mild and transient. Device-related serious adverse events (SAEs) have not been observed, either

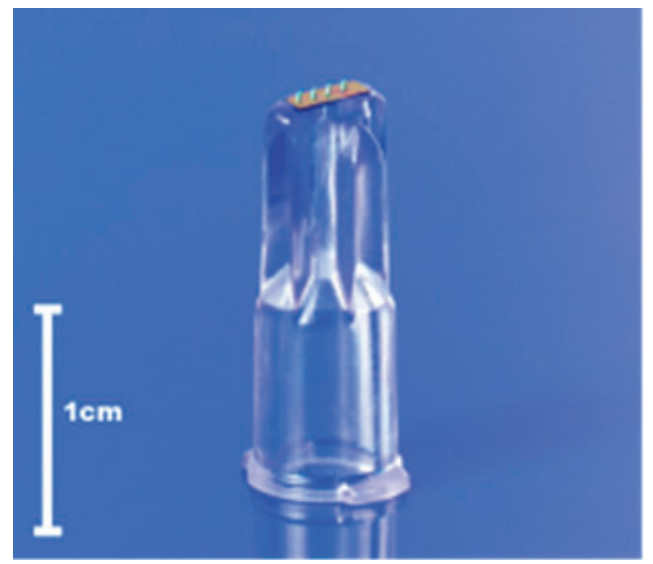

FIG. 2. The experimental MicronJet needle device. with the MJ needle or with its successor model, the MJ 600 needle, which has 3 microneedles of $0.6 \mathrm{~mm}$ length. ${ }^{14}$

This study was designed to assess the pharmacokinetic and safety profile of ID insulin delivered through an MJ needle compared with SC delivery of insulin in patients with type 2 diabetes. We report the ID delivery of Insulin aspart (Novorapid; Novo Nordisk) with the use of the novel MJ needle versus SC delivery while evaluating the relative safety and $\mathrm{PK} / \mathrm{PD}$ profile of the two insulin delivery methods in patients with type 2 diabetes.

\section{Research Design and Methods}

\section{Study oversight}

This was an open-label, single-center, pilot crossover study designed to evaluate the pharmacokinetic/pharmacodynamic (PK/PD) profile, safety, and tolerability of ID injection of aspart using MJ needle versus SC injection using NovoPen with a conventional needle in patients with type 2 diabetes. The study was performed in the Diabetes Unit, Division of Internal Medicine; Hadassah Medical Organization (Jerusalem, Israel). The study was approved by the Institutional Ethics Board. All subjects who participated in the study provided a signed informed consent form (NCT00602914).

\section{Study population}

Eligible patients had type 2 diabetes and were aged 30-70 years, with a body-mass index $(\mathrm{BMI})<35$, HbA1c of $6.5 \%-$ $10 \%$, and were treatment naïve or treated with metformin alone. Females of childbearing potential were not included. Exclusion criteria included hypersensitivity to any drug, any disease or condition known to interfere with the absorption, distribution, metabolism, or excretion of drugs, clinically significant medical disorders (heart, lung, liver, or kidney), history of recent alcohol or other substance abuse, or positive hepatitis B, hepatitis C, or HIV serologies.

\section{Study conduct}

The study included three groups of patients with type 2 diabetes, originally planned to be of equal size with six participants in each group (Table 1). Group 1 had subjects receiving two single injections of aspart (Novo Nordisk) $0.2 \mathrm{U} / \mathrm{kg}$, one ID using the MJ needle and one SC with NovoPen and a conventional needle. Injections were delivered in a randomized order to each individual before a standard meal. Group 2 had subjects receiving same regimen under fasting conditions. Group 3 had subjects receiving four single injections of aspart $0.2 \mathrm{U} / \mathrm{kg}$, two ID and two SC in a randomized order under fasting conditions. Injections were conducted 4-14 days apart. Subjects taking metformin regularly did not take it on study day.

The ID injections were done using MJ, a microneedle device comprising four microneedles, each $0.45 \mathrm{~mm}$ in length, with width comparable with a $\sim 30 \mathrm{G}$ needle, and mounted on a standard syringe. The NovoPen ${ }^{\circledR} 25 \mathrm{G} / 1^{\prime \prime}$ conventional steel needle mounted on Novopen served as the reference device. All injections were conducted in the right lower abdomen.

Blood samples for insulin and glucose were collected at the following times: $5 \mathrm{~min}$ before dose administration, at baseline, at 10 -min intervals between 0 and $2 \mathrm{~h}$, and at 30-min intervals between 2 and $6 \mathrm{~h}$ postdose administration. Insulin 
Table 1. Study Groups

\begin{tabular}{lccll}
\hline Group & $\begin{array}{c}\text { ITT } \\
\text { population }\end{array}$ & $\begin{array}{c}\text { PP } \\
\text { population }\end{array}$ & $\begin{array}{c}\text { Testing } \\
\text { condition }\end{array}$ & \multicolumn{1}{c}{ Treatment } \\
\hline 1 & 4 & 3 & Standard meal & Two single insulin injections: one with MJ and one with NovoPen \\
2 & 7 & 6 & Fasting & Two single insulin injections: one with MJ and one with NovoPen \\
3 & 6 & 5 & Fasting & Four single insulin injections: two with MJ and two with NovoPen \\
\hline
\end{tabular}

The trial included 17 patients in the ITT group, with 14 patients completing the study PP.

ITT, intention to treat; MJ, MicronJet; PP, per protocol.

levels were measured using the ADVIA Centaur XP Immunoassay System by Siemens. ${ }^{15}$

Local site reactions as well as any adverse events (AEs) or SAEs occurring on the day of study or on the subsequent visits were recorded, as well as their possible association with the intervention.

Tolerability endpoints were pain per visual analog scale (VAS) and a survey of subjects' preference parameters.

\section{Statistical analysis}

The study was designed as a pilot study and sample size determination was not planned to meet any specific significance and power requirements.

Descriptive statistics were calculated for all data. No imputation for missing values was applied. PK parameters (Tmax, Cmax, and area under the curve [AUC]) were derived from the individual insulin concentration data. The data were analyzed using SPSS software (version 20.01; SPSS, Inc.). PK analyses were carried out using PK Solutions 2.0 ${ }^{\mathrm{TM}}$. PK evaluations were carried out for each individual and the average results per group are displayed. Patients included in group 3 had two measurements for each injection type and the average of their two measurements was considered when calculating the group's average. PD data were derived from the blood glucose levels measured during the study. Comparison between groups was assessed using the Mann-Whitney test. Relative bioavailability (f) was calculated by comparing insulin kinetics between the investigational MJ device and standard SC Novopen. Within-subject comparison of tolerability of ID versus SC injections was carried out using the Wilcoxon paired test.

Post hoc analyses included assessment of the time to $50 \%$ insulin Cmax in each of the groups as well as measurement of partial AUC of insulin and glucose in the early (60 and $90 \mathrm{~min}$ ) and the late (4-6h) postinjection times.

Table 2. Patient Demographics

\begin{tabular}{lcc}
\hline & $\begin{array}{c}\text { ITT } \\
\text { population }\end{array}$ & $\begin{array}{c}\text { PP } \\
\text { population }\end{array}$ \\
\hline$N$ & 17 & 14 \\
Gender (male), $n(\%)$ & $16(94.1)$ & $13(92.9)$ \\
Age, years & $54.4 \pm 10.0$ & $55.7 \pm 9.1$ \\
Weight, $\mathrm{kg}$ & $85.2 \pm 7.3$ & $85.6 \pm 7.6$ \\
$\mathrm{BMI}, \mathrm{kg} / \mathrm{m}^{2}$ & $28.4 \pm 3.6$ & $28.4 \pm 3.3$ \\
Diabetes duration, years & $6.8 \pm 4.6$ & $7.21 \pm 4.5$ \\
Concomitant metformin, $n(\%)$ & $8(47.1)$ & $6(42.9)$ \\
\hline
\end{tabular}

Data are mean \pm SD.

BMI, body-mass index.

\section{Results}

\section{Patient characteristics}

Seventeen subjects were enrolled and 14 completed the study per-protocol and are included in all further analyses. One patient was excluded from the trial due to protocol violationHbAlc of $5.84 \%$ and BMI $>35 \mathrm{~kg} / \mathrm{m}^{2}$, one dropped out by personal choice, and one was excluded as per investigator's decision following a hypoglycemic AE.

Table 2 lists demographics of all patients recruited to the trial and of those completing the trial per-protocol.

\section{Pharmacokinetics and pharmacodynamics}

ID insulin injection with the MJ needle resulted in a shorter Tmax than the SC injection (Table 3). Interpatient variability in Tmax was lower with ID versus SC injections in the overall population (inter quartile range/median $28.6 \%$ vs. $62.9 \%$ ). Higher insulin Cmax values were observed with ID versus SC injection, yet this did not reach statistical significance (Table 3). Post hoc analysis of time to 50\% Cmax was significantly shorter with the ID versus the SC injections (median 14.0 vs. $26.0 \mathrm{~min}, p=0.008$ ). The PK profile of insulin injections, ID versus SC, is presented in Figure 3.

Post hoc analysis revealed that the insulin AUC 60 and 90 min after insulin administration were significantly higher in ID versus SC injection; 4-6h after insulin injection, the AUC in the ID group was significantly lower than SC insulin injection (Table 4).

The relative bioavailability of the ID versus the SC insulin $\left(\mathrm{AUC}_{\mathrm{ID}} / \mathrm{AUC}_{\mathrm{SC}}\right.$ ) was similar (median 0.91 [95\% confidence interval 0.73-1.27]).

Pharmacodynamic data of the glucose levels measured under fasting conditions are presented in Figure 4. The glucose AUC during 4-6h postinjection was lower in the ID injection versus the SC injection (Table 4).

\section{AEs and tolerability}

All 17 patients recruited to the trial were included in the safety and tolerability analysis. No local AEs (injection site reactions) were reported. A total of 3 of 17 subjects experienced 5 AEs. Three subjects experienced mild hypoglycemia, which resolved with oral glucose consumption. One of them had glucose levels of $40 \mathrm{mg} / \mathrm{dL}$ at $80 \mathrm{~min}$ following his first SC injection, which was followed by a slight increase to $58 \mathrm{mg} / \mathrm{dL}$ at $120 \mathrm{~min}$. The subject was withdrawn from the study by the investigator's decision. One additional patient noted acute gastroenteritis one day following the ID injection, with subsequent anxiety, and elected to withdraw from the study. 
Table 3. Insulin Kinetics by Treatment Group

\begin{tabular}{|c|c|c|c|c|c|c|c|c|}
\hline \multirow[b]{2}{*}{ Data } & \multicolumn{3}{|c|}{ Fasting $(\mathrm{n}=11)$} & \multicolumn{2}{|c|}{ Standard meal test $(\mathrm{n}=3)$} & \multicolumn{3}{|c|}{ All patients $(\mathrm{n}=14)$} \\
\hline & $I D$ & $S C$ & $\mathrm{P}$ & $I D$ & $S C$ & $I D$ & $S C$ & $\mathrm{P}$ \\
\hline $\begin{array}{l}\text { Tmax, Min } \\
\text { Median } \\
\text { IQR }\end{array}$ & $\begin{array}{l}\text { utes } \\
\quad 35 \\
\quad 30-40\end{array}$ & $\begin{array}{c}90 \\
60-115\end{array}$ & $<0.001$ & $\begin{array}{c}50 \\
30-60\end{array}$ & $\begin{array}{c}70 \\
50-90\end{array}$ & $\begin{array}{c}35 \\
30-40\end{array}$ & $\begin{array}{c}87.5 \\
60-110\end{array}$ & $<\mathbf{0 . 0 0 1}$ \\
\hline $\begin{array}{c}\mathrm{Cmax}, \mu \mathrm{U} / \\
\text { Median } \\
\mathrm{IQR}\end{array}$ & $\begin{array}{c}\mathrm{nL} \\
79 \\
47-104\end{array}$ & $\begin{array}{c}54.5 \\
46-58\end{array}$ & 0.125 & $\begin{array}{c}95 \\
78-98\end{array}$ & $\begin{array}{c}68 \\
64-111\end{array}$ & $\begin{array}{c}80 \\
51-98\end{array}$ & $\begin{array}{c}55 \\
47-68\end{array}$ & 0.085 \\
\hline $\begin{array}{l}\text { AUC, } \mu \mathrm{U} / \mathrm{n} \\
\text { Median } \\
\text { IQR }\end{array}$ & $\begin{array}{l}\mathrm{L} L / \min \\
\quad 9672.5 \\
5578-12125\end{array}$ & $\begin{array}{c}10,407.5 \\
9280-12196\end{array}$ & 0.215 & $\begin{array}{c}12690 \\
10060-17655\end{array}$ & $\begin{array}{c}13960 \\
12640-23050\end{array}$ & $\begin{array}{c}9913.75 \\
5800-13685\end{array}$ & $\begin{array}{c}10936.25 \\
9305-13690\end{array}$ & 0.077 \\
\hline
\end{tabular}

Fourteen patients with diabetes received injections of aspart (Novorapid), both ID using a MicronJet needle and SC injections, in a crossover design. Eleven were in the fasting state and 3 received the injection before a standard meal test. Insulin pharmacokinetics is shown. $P$ value was not calculated in the subjects receiving insulin poststandard meal test due to the small number of subjects.

ID, intradermal; SC, subcutaneous.

$P$ values in boldface are statistically significant.

Pain evaluation by VAS on a range of $1-100$ was done for both insertion and injection pain. There was no statistical significant difference in insertion pain between the ID and the SC injections (mean \pm SD: $8.97 \pm 9.97$ and $6.84 \pm 4.76$, respectively, $p=0.975$ ). Greater injection pain was noted with the ID injection versus the SC injection (mean \pm SD: $15.78 \pm 15.05$ and $4.14 \pm 4.77$, respectively, $p=0.023$ ). Patients in group 3 who received two injections of each type noted reduced injection pain in the second ID injection compared with the first (mean \pm SD: $12.2 \pm 10.6$ vs. $4.5 \pm 3.8$, $p=0.043$ ). Overall, use of the MJ device was associated with minimal discomfort, with the highest VAS score recorded lower than the 50\% threshold. In a subject preference survey, answered by the 14 patients who completed the study PP, 7 patients said the MJ technology enabled painless injection, 4 were neutral, and 3 disagreed. Five patients said they would prefer the ID injection in the future, eight were neutral, and one preferred the SC injection.

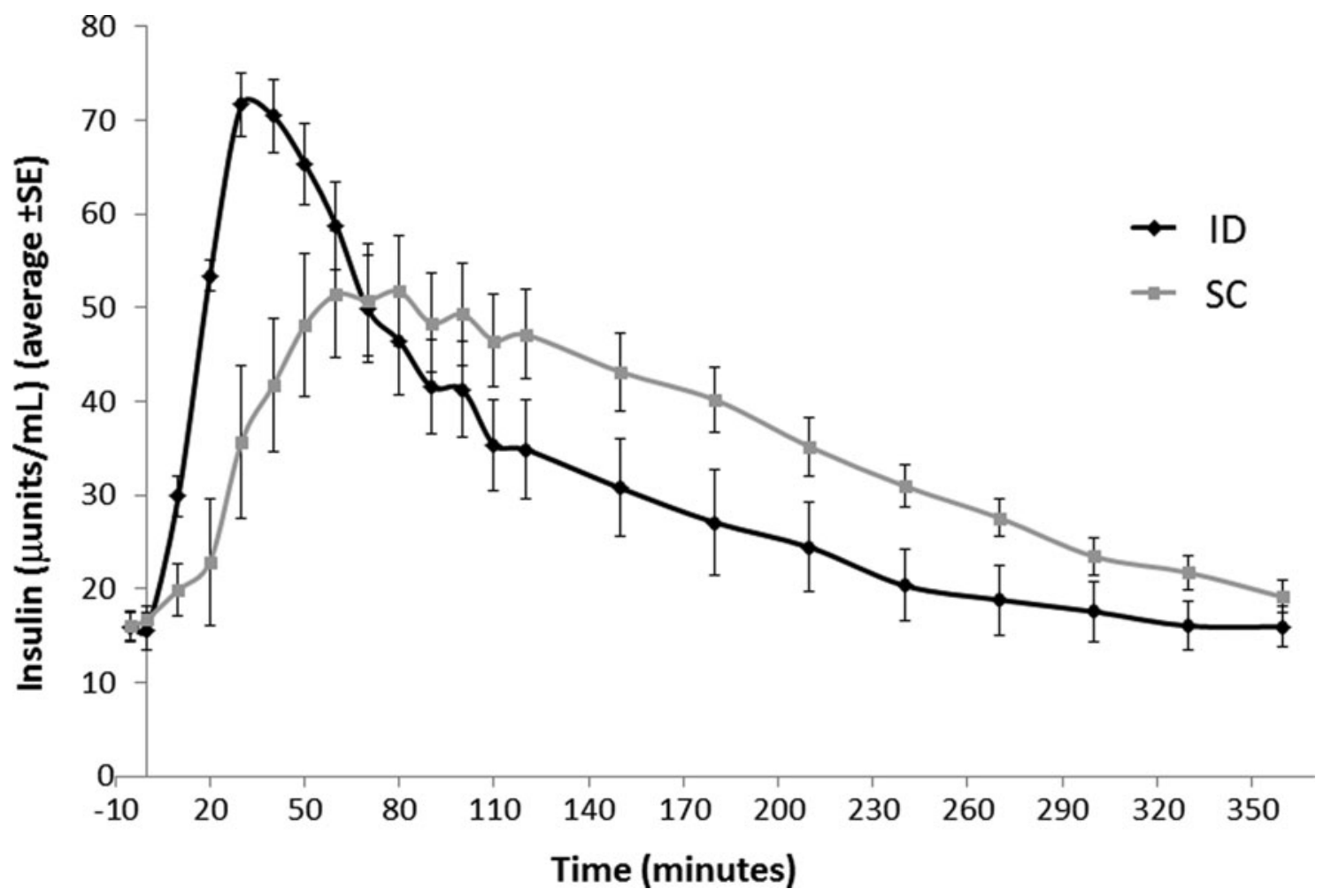

FIG. 3. PK profile of insulin: Fourteen patients with T2DM were injected $0.2 \mathrm{U} / \mathrm{kg}$ aspart using an intradermal (ID) route through a MicronJet (MJ) needle or subcutaneous (SC) route using a standard needle. Three patients were injected before a standard meal test and five patients received two injections of each type. All injections were delivered in a random order, with 4-14 days between injections. The plasma insulin levels during the $6 \mathrm{~h}$ following the injections are displayed. Each curve represents the average of 14 patients. 
Table 4. Partial Insulin and Glucose Area Under The Curve Analysis

\begin{tabular}{lcc}
\hline & Intradermal (MicronJet) & Subcutaneous \\
\hline Overall population $(n=14)$ & & \\
Insulin AUC 0-1 & $3820.9 \pm 1428.6$ & $2534.1 \pm 737.1$ \\
Insulin AUC 0-1.5 & $5156.3 \pm 1988.7$ & $4035.4 \pm 1255.9$ \\
Insulin AUC 4-6 & $2054.4 \pm 857.7$ & $2929.0 \pm 1412.1$ \\
Fasting population $(n=11)$ & $3695.2 \pm 1593.3$ & \\
Insulin AUC 0-1 & $4912.0 \pm 2154.6$ & $2346.1 \pm 609.7$ \\
Insulin AUC 0-1.5 & $2027.3 \pm 946.5$ & $3647.7 \pm 904.0$ \\
Insulin AUC 4-6 & & $2903.3 \pm 1571.9$ \\
Fasting population $(n=11)$ & $9295.8 \pm 2772.6$ & $\mathbf{0 . 0 1}$ \\
Glucose AUC 0-1 & $12,352.4 \pm 3943.6$ & $9713.0 \pm 2169.3$ \\
Glucose AUC 0-1.5 & $12,125.2 \pm 2405.2$ & $12,814.8 \pm 2664.8$ \\
Glucose AUC 4-6 & $9908.9 \pm 1555.2$ & $\mathbf{0 . 0 2 9}$ \\
\hline
\end{tabular}

Fourteen patients with diabetes received injections of aspart (Novorapid), both ID using a MicronJet needle and SC injections, in a crossover design. Eleven were in the fasting state and 3 received the injection before a standard meal test. Partial AUC data are shown for insulin $(\mu \mathrm{U} / \mathrm{mL} / \mathrm{min})$ and glucose $(\mathrm{mg} \% / \mathrm{min})$ in the early (first 1 and $1.5 \mathrm{~h}$ postinjection) and late (4-6h postinjection) phases. Data are mean $\pm \mathrm{SD}$.

AUC, area under the curve.

$P$ values in boldface are statistically significant.

\section{Discussion}

This study demonstrates the superior pharmacological profile of ID injection of aspart with the MJ needle versus SC injection of the same insulin utilizing a conventional needle. The ID delivery of insulin demonstrated a shorter Tmax, higher early exposure, and reduced interpatient variability in Tmax. Additionally, time to 50\% Cmax was significantly shorter with ID versus SC injection. Finally, late AUC glucose levels were higher with ID injection, potentially limiting late hypoglycemic events.

The ID injection of insulin by the MJ device yielded a good safety profile, with no significant additional risk compared with conventional SC administration.

ID delivery of insulin to accelerate its absorption into the systemic circulation has been evaluated in several clinical studies

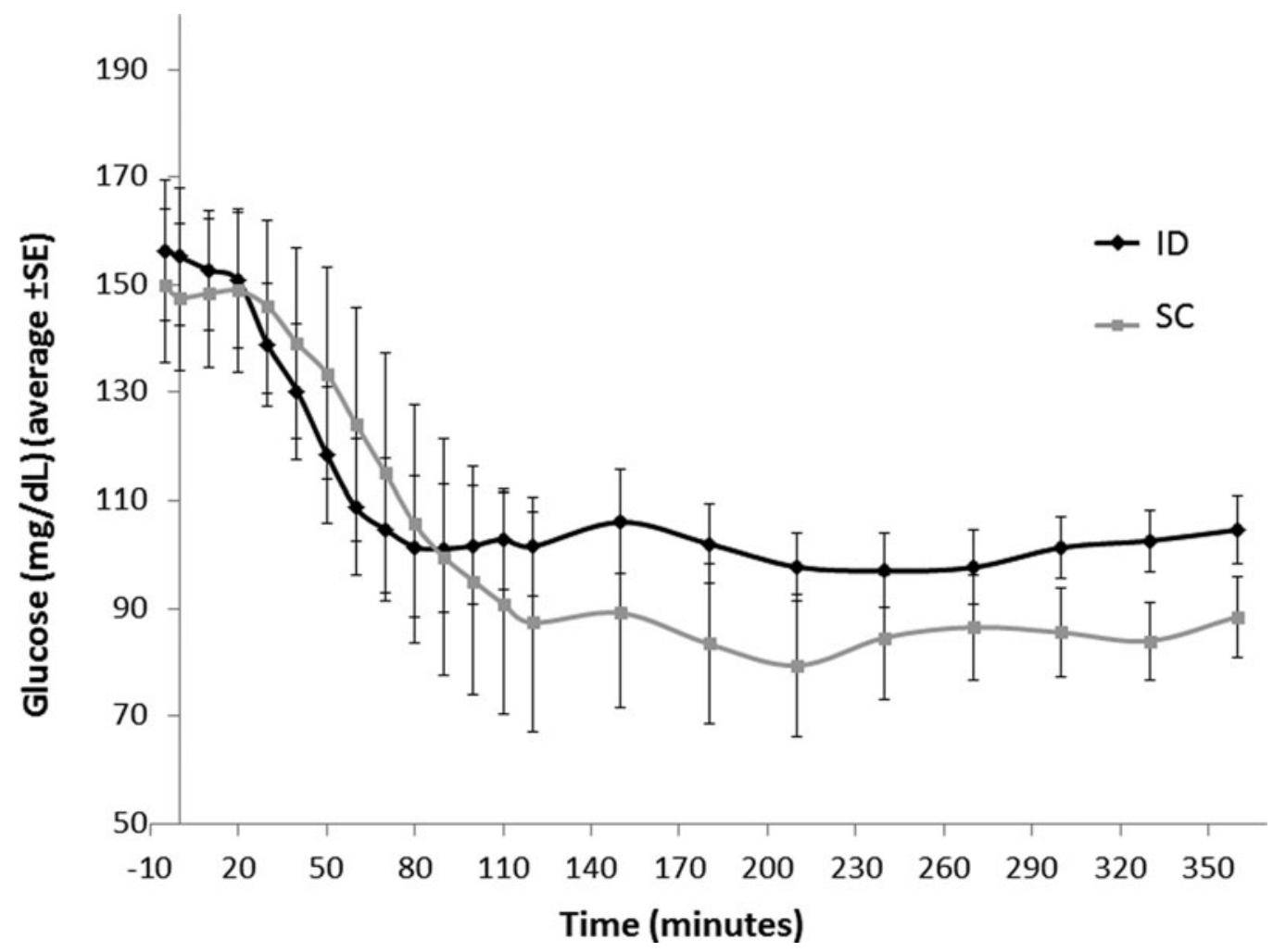

FIG. 4. PD profile of glucose in fasting patients: Legend: Eleven patients with T2DM were injected $0.2 \mathrm{U} / \mathrm{kg}$ aspart using the ID route through a MicronJet (MJ) needle or SC route using a standard needle and remained fasting for $6 \mathrm{~h}$ following the injection. Five patients received two injections of each type. All injections were delivered in a random order, with 4-14 days between injections. The plasma glucose levels during the $6 \mathrm{~h}$ following the injections are displayed. Each curve represents the average of 11 patients. 
of healthy volunteers or patients with type 1 diabetes. ${ }^{16-23}$ Gupta et al. demonstrated that ID insulin administration through microneedle reached peak insulin concentrations in approximately half of the time than the catheters, resulting in a better reduction of plasma glucose levels. ${ }^{16-17}$ ID administration of Insulin Lispro or regular human insulin by microneedles showed significantly faster uptake and time to maximum concentration, higher maximum concentration, and shorter systemic circulating duration versus SC application, both in healthy male volunteers or type 1 diabetes mellitus (T1DM) patients. ${ }^{18-19}$ In children and adolescents with T1DM, it was found that insulin onset and offset time (defined as time to $50 \%$ Cmax during insulin onset [T 50\% max rising] and offset [T 50\% max falling]) was faster after microneedle delivery compared with SC delivery, and the pain was significantly lower. ${ }^{20}$ ID delivery of insulin demonstrated safety and efficacy in continuous infusion through a microneedle-based continuous insulin infusion pump. ${ }^{21}$

The Tmax of the SC injection in our trial was longer compared with previously reported studies. Tmax of the SC insulin in our study was $87.5 \mathrm{~min}$ compared with $51.6 \mathrm{~min}$ measured by McVey et al. ${ }^{22}$ and 57 min measured by Gupta et al. ${ }^{17}$ The Tmax of the ID injection in the three trials was 35, 36, and 27 min, respectively. Slower absorption of subcutaneous insulin in patients with type 2 diabetes versus type 1 diabetes has been reported, ${ }^{24}$ and further studies in patients with type 2 diabetes will be needed to further support this observation.

The importance of mitigating postprandial glucose excursion and minimizing glycemic variability has been extensively discussed in patients with type 1 and type 2 diabetes. ${ }^{25}$ Multiple approaches to expedite insulin delivery in patients with type 2 diabetes to minimize postprandial glucose excursions are being explored. ${ }^{5}$ Our study is the first to demonstrate the superior PK and PD profile of ID insulin in patients with type 2 diabetes, creating a safe and welltolerated insulin delivery mode for this population, which carries potentially better postprandial glucose control and lower risk of hypoglycemia.

Our study carries several limitations. The dose of insulin administered was small, and patients with type 2 diabetes may often require higher doses of insulin to attain glycemic control. Further study with higher insulin doses and a doseresponse study of ID absorption of insulin at higher doses are warranted. Additionally, intrasubject variability was not assessed due to the small number of patients receiving more than one injection of each type and only two injections. Furthermore, the insulin assay used detected to a similar extent human insulin and aspart. Finally, the trial included only a small number of subjects tested postprandially, which did not allow for full statistical analysis comparing the postprandial glucose PD between the ID and SC groups.

In conclusion, the PK profile of ID insulin delivery by MJ in patients with type 2 diabetes is improved - reaching earlier systemic insulin levels, lower late insulin levels, and higher late glucose levels, potentially reducing the risk for postprandial hypoglycemia. The lack of significant safety, convenience, or tolerability issues supports the use of the MJ needle in patients with diabetes.

\section{Acknowledgment}

This study was sponsored by NanoPass Technologies Ltd.

\section{Disclosure Statement}

I.R. reports the following: Advisory Board: AstraZeneca/ Bristol-Meyers Squibb, Eli Lilly and Company, Medscape LLC, Merck Sharp \& Dohme Limited, Novo Nordisk, Inc., Sanofi, Orgenesis, SmartZyme Innovation Ltd, and Labstyle Innovations Ltd; Consultant: AstraZeneca/Bristol-Myers Squibb, Insuline Medical, Gili Medical, Kamada Ltd., and FuturRx Ltd.; Speaker's Bureau: AstraZeneca/Bristol-Myers Squibb, Eli Lilly and Company, Johnson \& Johnson, Merck Sharp \& Dohme Limited, Novartis Pharma AG, Novo Nordisk, Inc., Sanofi, and Teva; Stock/Shareholder: Insuline Medical, Labstyle Innovations SmartZyme Innovation Ltd., Orgenesis, and Glucome Ltd.

A.C. reports receipt of consulting fees and payment for lectures from AstraZeneca, Boehringer Ingelheim, Elli Lilly, MSD, Novartis, Novo Nordisk, and Sanofi.

E.K. and Y.L. are employed by NanoPass Technologies.

\section{References}

1. Cefalu WT, Rosenstock J, LeRoith D, et al.: Insulin's role in diabetes management: after 90 years, still considered the essential "black dress." Diabetes Care 2015;38:2200-2203.

2. Home PD: The pharmacokinetics and pharmacodynamics of rapid-acting insulin analogues and their clinical consequences. Diabetes Obes Metab 2012;14:780-788.

3. Heinemann L, Muchmore DB: Ultrafast-acting insulins: state of the art. J Diabetes Sci Technol 2012;6:728-742.

4. Cengiz E: Closer to ideal insulin action: ultra fast acting insulins. Panminerva Med 2013;55:269-275.

5. Cahn A, Miccoli R, Dardano A, et al.: New forms of insulin and insulin therapies for the treatment of type 2 diabetes. Lancet Diabetes Endocrinol 2015;3:638-652.

6. Carr TF, Saltoun CA: Skin testing in allergy. Allergy Asthma Proc 2012;33: S6-S8.

7. Al-Jahdali H, Memish ZA, Menzies D: The utility and interpretation of tuberculin skin tests in the Middle East. Am J Infect Control 2005;33:151-156.

8. Flynn PM, Shenep JL, Mao L, et al.: Influence of needle gauge in Mantoux skin testing. Chest J 1994;106:1463-1465.

9. Tarnow K, King N: Intradermal injections: traditional bevel up versus bevel down. Appl Nurs Res 2004;17:275-282.

10. Van DP, Oosterhuis-Kafeja F, Van der WM, et al.: Safety and efficacy of a novel microneedle device for dose sparing intra-dermal influenza vaccination in healthy adults. Vaccine 2009;27:454-549.

11. Hung IF, Levin Y, To KK: Quantitative and qualitative analysis of antibody response after dose sparing intradermal 2009 H1N1 vaccination. Vaccine 2012;30:2707-2708.

12. Hung IF, Levin Y, To KK, et al.: Dose sparing intradermal trivalent influenza (2010/2011) vaccination overcomes reduced immunogenicity of the 2009 H1N1 strain. Vaccine 2012; 30:6427-6435.

13. Levin Y, Kochba E, Kenney R: Clinical evaluation of a novel microneedle device for intradermal delivery of an influenza vaccine: are all delivery methods the same? Vaccine 2014;32:4249-4252.

14. Levin Y, Kochba E, Hung I, et al.: Intradermal vaccination using the novel microneedle device MicronJet600: past, present, and future. Hum Vaccin Immunother 2015;11:991-997.

15. Parfitt C, Church D, Armston A, Couchman L, Evans C, Wark G, McDonald TJ. Commercial insulin immunoassays fail to detect commonly prescribed insulin analogues. Clin Biochem 2015;48:1354-1357. 
16. Gupta J, Felner EI, Prausnitz MR: Minimally invasive insulin delivery in subjects with type 1 diabetes using hollow microneedles. Diabetes Technol Ther 2009;11:329-337.

17. Gupta J, Felner EI, Prausnitz MR: Rapid pharmacokinetics of intradermal insulin administered using microneedles in type 1 diabetes subjects. Diabetes Technol Ther 2011;13: 451-456.

18. Pettis RJ, Ginsberg B, Hirsch L, et al.: Intradermal microneedle delivery of insulin lispro achieves faster insulin absorption and insulin action than subcutaneous injection. Diabetes Technol Ther 2011;13:435-442.

19. Pettis RJ, Hirsch L, Kapitza C, et al.: Heinemann L. Microneedle-based intradermal versus subcutaneous administration of regular human insulin or insulin lispro: pharmacokinetics and postprandial glycemic excursions in patients with type 1 diabetes. Diabetes Technol Ther 2011; 13:443-450.

20. Norman JJ, Brown MR, Raviele NA, et al.: Faster pharmacokinetics and increased patient acceptance of intradermal insulin delivery using a single hollow microneedle in children and adolescents with type 1 diabetes. Pediatr Diabetes 2013;14:459-465.

21. Rini CJ, McVey E, Sutter D, et al.: Intradermal insulin infusion achieves faster insulin action than subcutaneous infusion for 3-day wear. Drug Deliv Transl Res 2015;5: 332-345.

22. McVey E, Hirsch L, Sutter DE, et al.: Pharmacokinetics and postprandial glycemic excursions following insulin lispro delivered by intradermal microneedle or subcutaneous infusion. J Diabetes Sci Technol 2012;6:743-754.

23. Engwerda EE, Abbink EJ, Tack CJ, et al.: Improved pharmacokinetic and pharmacodynamic profile of rapid-acting insulin using needle-free jet injection technology. Diabetes Care 2011;34:1804-1808.

24. Clauson PG, Linde B. Absorption of rapid-acting insulin in obese and nonobese NIDDM patients. Diabetes Care 1995;18: 986-991.

25. Hirsch IB: Glycemic variability and diabetes complications: does it matter? of course it does! Diabetes Care 2015; $38: 1610-1614$.

Address correspondence to: Efrat Kochba, MD

NanoPass Technologies Ltd. 3 Golda Meir Street Nes Ziona 7403648

Israel

E-mail: efrat@nanopass.com 